\title{
NanoFab with SIMS - Recent Results from the BAM-L200 Analytical Standard and Semiconductor Samples
}

\author{
Brett Lewis ${ }^{1}$, Fouzia Khanom ${ }^{1}$, and John Notte ${ }^{1}$ \\ 1. Carl Zeiss Microscopy, One Corporation Way, Peabody, MA 01960, USA
}

The ZEISS ORION NanoFab is a multi-ion beam platform which makes available a combination of helium, neon, and gallium focused ion beams (FIBs). The helium beam is commonly used for imaging, and in this capacity reveals sub-nanometer details with high contrast, surface sensitivity, minimal charging artifacts, and a long depth of focus. The gallium ion beam is used for traditional FIB applications, providing very high sputter rates for bulk material removal such as applications like lamella preparation. The neon beam with its intermediate mass provides an appreciable sputter yield with higher precision and reduced implantation effects. Together, this combination of ion beams have enabled many diverse, cutting-edge nanofabrication tasks such as resist lithography, precision sputtering, beam-assisted chemistry, and dislocation engineering [1].

While there are a handful of analytical techniques based on ion beams in this energy range, one of the most favored is secondary ion mass spectrometry (SIMS). Conventional SIMS instruments are limited by focused probe sizes larger than $50 \mathrm{~nm}$, due to the low brightness of their ion sources. In contrast, NanoFab's neon beam can be focused to under $2 \mathrm{~nm}$, enabling analysis of regions limited only by the lateral distribution of the surface sputtered atoms [2,3]. In light of this advantageous opportunity, the NanoFab has recently been equipped with a magnetic spectrometer to permit SIMS analysis. The magnetic spectrometer has demonstrated an extraction and transmission efficiency of about $40 \%$, and the mass resolution $(\mathrm{M} / \Delta \mathrm{M})$ of about 500 .

To demonstrate the lateral resolution of the ZEISS ORION NanoFab with SIMS, we have used the wellestablished BAM-L200 standard [4] to characterize the fidelity and acuity of the elemental mapping. The BAM-L200 is a certified reference for the calibration and testing of the lateral resolution for a variety of analytical techniques. With the SIMS detectors configured for aluminum and gallium, the spectrometer produced the complementary images of stripes (alternately GaAs and AlGaAs) as shown in Fig 1a and 1b, and shown as a composite colorized image in Fig 1c. With a $14 \mu \mathrm{m}$ FOV, these images were acquired with a $25 \mathrm{keV}$ neon beam with $9.5 \mathrm{pA}$ of probe current and a $300 \mu \mathrm{s}$ dwell time. When imaged at higher magnifications (e.g. $2 \mu \mathrm{m} \mathrm{FOV}$ ), the finest line pairs with a half pitch of $9 \mathrm{~nm}$ are readily recognized.

As a pertinent application, we present SIMS images from a semiconductor device based on $22 \mathrm{~nm}$ finFETs. The device has been previously de-processed so that multiple layers with different compositions can be simultaneously imaged. Figure 2 shows a $10 \mu \mathrm{m}$ FOV region of this sample with features as small as 50 $\mathrm{nm}$ visible. The focused helium beam was used to acquire Figure 2a which is based on the generation of secondary electrons and provides sub-nanometer resolution. Figure $2 b$ was acquired with the SIMS spectrometer, unambiguously revealing different elemental compositions within each layer. Figure 2c shows the advantage of correlative microscopy. The best aspects of the two previous images, namely best resolution and best elemental composition, are combined to produce a correlative image acquired from a single instrument (Fig 2c). 

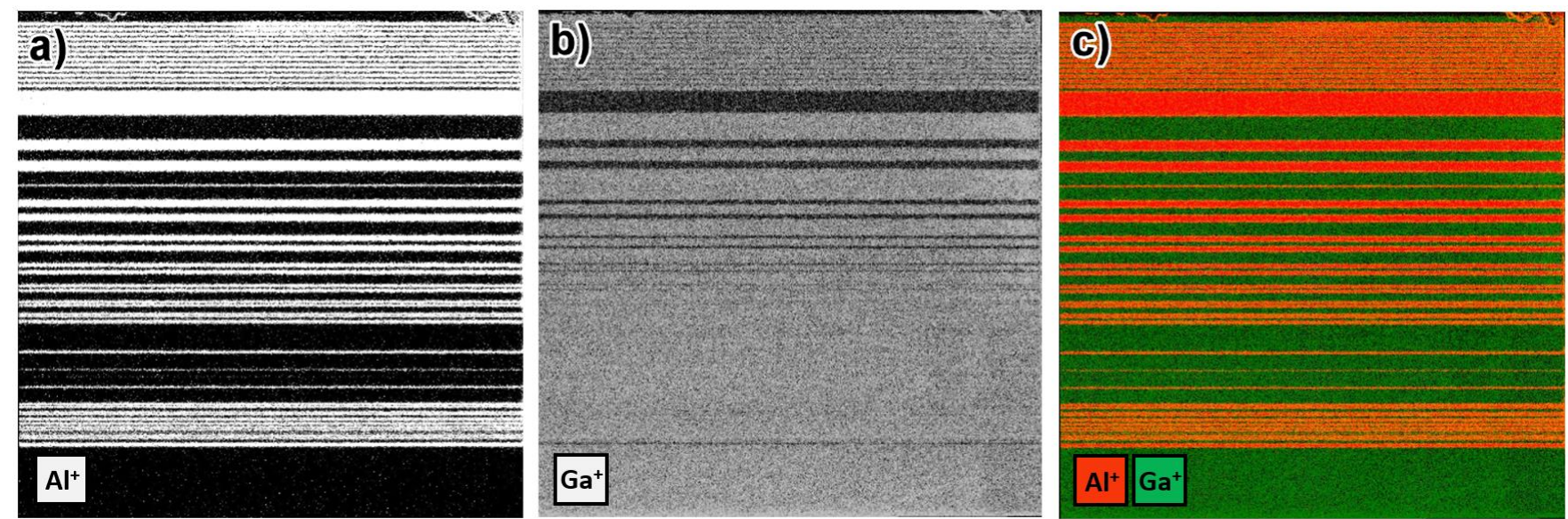

Figure 1: Zeiss ORION NanoFab SIMS generated elemental mapping images of the BAM-L200 standard. The images in (a) and (b) show the images acquired with detection set for aluminum and gallium. Image (c) is a composite image with the two elements colorized as shown.
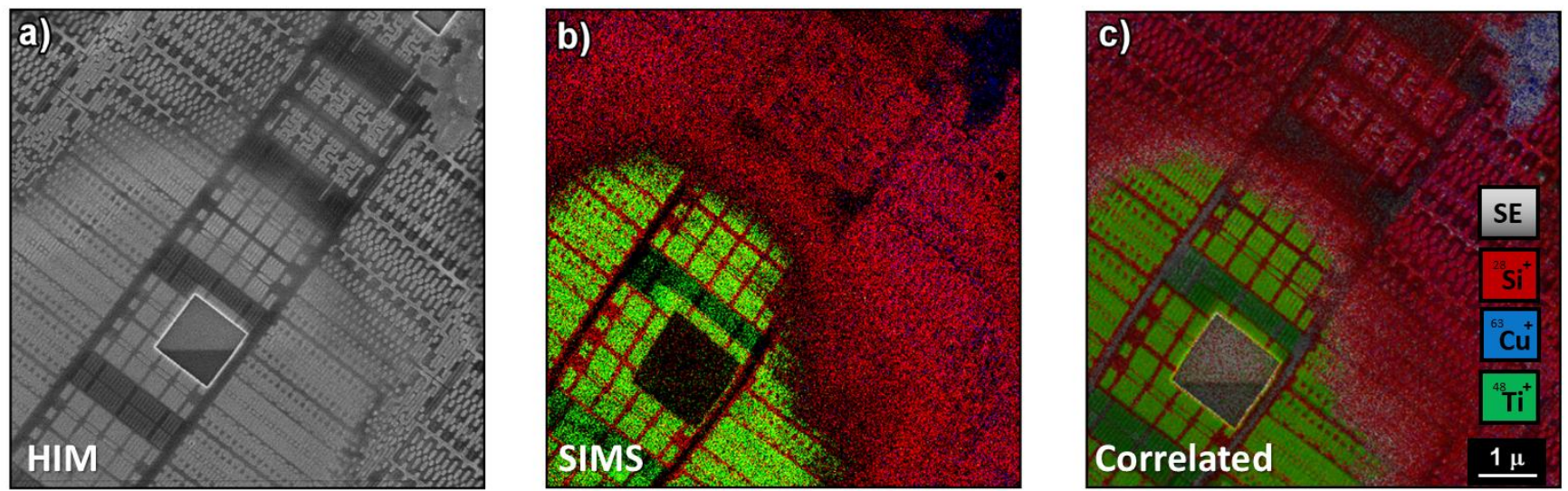

Figure 2: a) $\mathrm{He}^{+}$ion produced secondary electron image b) SIMS elemental mapping image c)

Correlated image with SIMS overlay. The elements mapped with SIMS are red (silicon), blue (copper), green (titanium) as labeled.

References:

[1] G. Hlawacek, A. Gölzhäuser, Helium Ion Microscopy, Springer, 2017

[2] T. Wirtz, D. Dowsett, P. Philipp, Helium Ion Microscopy, edited by G. Hlawacek, A. Gölzhäuser, Springer, 2017

[3] T. Wirtz, P. Philipp, J.-N. Audinot, D. Dowsett, S. Eswara, Nanotechnology 26 (2015) 434001

[4] Bundesanstalt für Materialforschung und -prüfung (BAM).

https://rrr.bam.de/RRR/Content/EN/Downloads/RM-certificates/RM-cert-layer-and-

surface/bam_1200.html 